\author{
I. A. Clemetson \\ A. Popp \\ K. Lippuner \\ F. Ballmer \\ S. E. Anderson
}

\section{Postpartum osteoporosis associated with proximal tibial stress fracture}

Received: 18 July 2003

Revised: 27 October 2003

Accepted: 28 October 2003

Published online: 9 January 2004

(C) ISS 2004

I. A. Clemetson $(\bullet) \cdot$ S. E. Anderson Department of Radiology,

University Hospital of Bern, Inselspital,

3010 Bern, Switzerland

e-mail: isabelle.clemetson@insel.ch

Tel.: +41-31-6322527

Fax: +41-31-6324874

A. Popp - K. Lippuner

Department of Osteology,

University Hospital of Bern, Inselspital,

3010 Bern, Switzerland

F. Ballmer

Knee and Sports Medicine Unit,

Lindenhofspital Bern,

3012 Bern, Switzerland

\begin{abstract}
A 33-year-old woman presented with acute nonspecific knee pain, 6 months postpartum. MR imaging, computed tomography and radiography were performed and a proximal tibia plateau insufficiency fracture was detected. Bone densitometry demonstrated mild postpartum osteoporosis. To our knowledge these findings have not been described in this location and in this clinical setting. The etiology of the atraumatic fracture of the tibia is presumed to be due to a low bone mineral density. The bone loss was probably due to pregnancy, lactation and postpartum hormonal changes. There were no other inciting causes and the patient was normocalcemic.
\end{abstract}

We discuss the presence of a postpartum stress fracture in a hitherto undescribed site in a patient who had lactated following an uncomplicated pregnancy and had no other identifiable cause for a stress fracture.

Keywords Tibial insufficiency fracture - Postpartum osteoporosis . Dual-energy X-ray absorptiometry . MR imaging of the knee .

Radiography

\section{Introduction}

Insufficiency fractures associated with postpartum osteoporosis are well known though uncommon. Previous sites of insufficiency fractures related to pregnancy and postpartum state described in the literature include sacrum [1, 2, 3]; superior and inferior pubic bones, ischiopubic arch and pubic body [4]; proximal femoral diaphysis, neck of femur and subcapital hip region $[5,6]$; rib $[7,8]$ and vertebrae [8]. Sports stress fractures are well documented in the tibia, pelvis, fibula, humerus and 2nd, 3rd and 4th metatarsal bones [9]. Risk factors of postpartum fracture are thought to be rapid and excessive weight gain during pregnancy $[1,4]$, transient osteopenia/ osteoporosis during pregnancy $[2,6,8,10]$, increase in physical activity such as jogging and aerobics during and immediately after pregnancy $[1,11]$, cesarian section or assisted vaginal delivery $[11,12]$, muscular forces acting on ribs during the enlargement of the uterus [7] and endocrine and hormonal influences [13, 14, 15]. None of these causes were present in our patient, who developed a tibial stress fracture 6 months postpartum, at the end of her lactation period.

\section{Case report}

The patient gave full written informed consent for publication of her history and imaging details.

A 33-year-old woman presented with nonspecific acute knee pain, 6 months postpartum. Her pregnancy was uncomplicated. She had no massive weight gain and did not exercise excessively during or after the pregnancy. In particular there was no history of trauma, excessive walking or cycling. Apart from a small daily walk with the new baby, accompanied by her two other children, she had normal daily activities. She was at the end of her nursing period (a total of 5 months) and there was no history of fracture or a family history of bone disease. There was no use of steroid medication or 

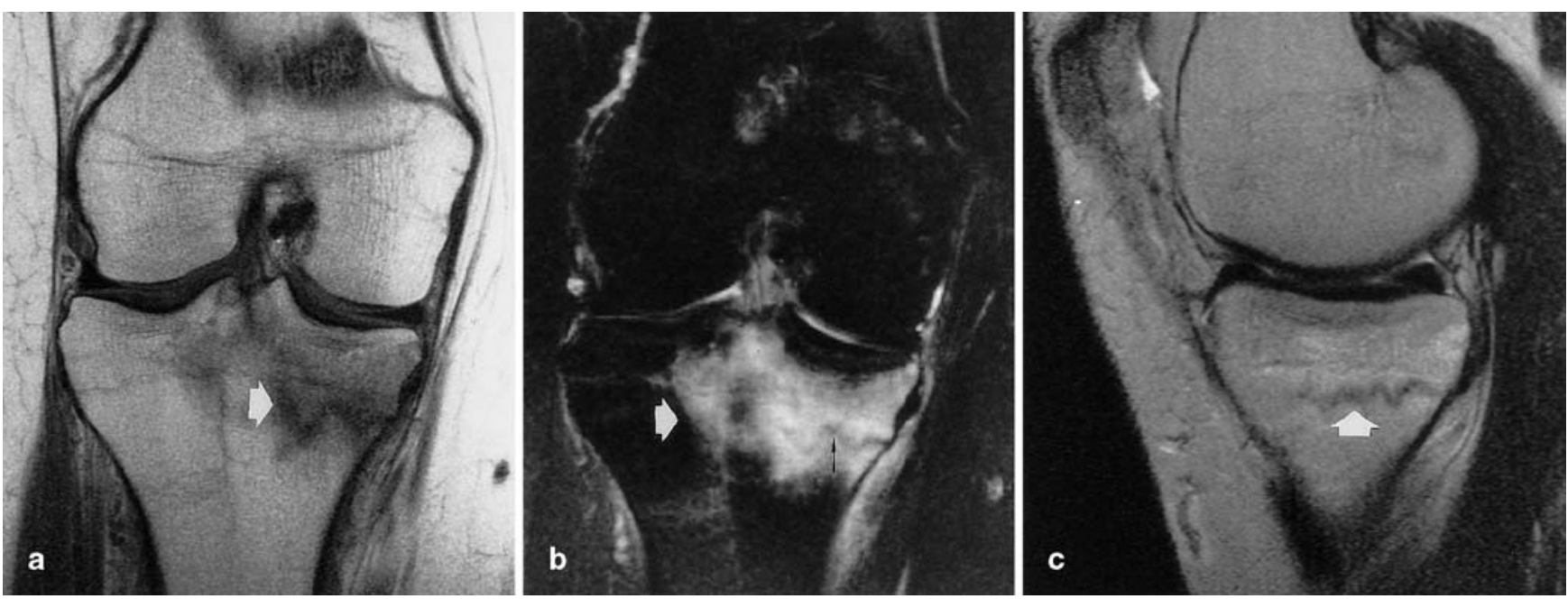

Fig. 1 A Coronal T1-weighted MR image (TR: 560, TE: 15) shows a medial tibial plateau insufficiency fracture (arrow). B Coronal STIR image (Short Tau Inversion Recovery) (Ti: 180, TR: 4200, TE: 96) shows markedly altered bone marrow signal in the

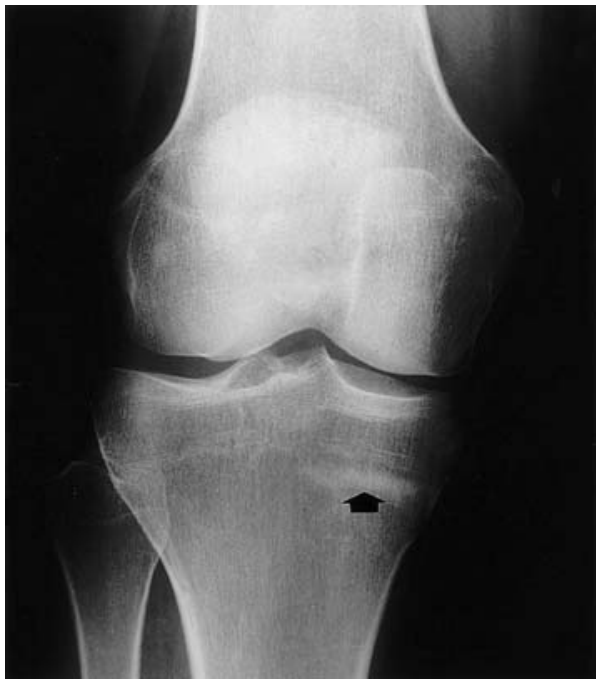

Fig. 2 Frontal radiograph of the right knee 3 months after the onset of acute atraumatic knee pain with an area of sclerosis in the proximal medial tibial plateau (arrow)

excessive alcohol intake reported. As the patient had no history of trauma or of overuse, dual-energy X-ray absorptiometry (DXA) was performed (Hologic QDR 4500c, Hologic, Bedford, Mass.). Low bone mineral density (BMD) was found at the lumbar spine (L3: $0.954 \mathrm{~g} / \mathrm{cm}^{2}, T$-score $-1.2 \mathrm{SD}, Z$-score $-1.2 \mathrm{SD}$ ), the contralateral femoral neck $\left(0.727 \mathrm{~g} / \mathrm{cm}^{2}, T\right.$-score $-1.1 \mathrm{SD}, Z$-score $-0.9 \mathrm{SD})$ and distal tibial epiphysis $\left(0.623 \mathrm{~g} / \mathrm{cm}^{2}, T\right.$-score $-1.5 \mathrm{SD}$, Z-score $-1.3 \mathrm{SD}$ ). Metabolic investigation showed diminished urinary calcium, normal blood calcium levels (ionized and total), a blood phosphate concentration at the upper limit of normal, and normal levels of 25-hydroxyvitamin D, parathyroid hormone (intact $\mathrm{PTH})$, osteocalcin and a normal urinary deoxypyridinoline/creati- proximal medial tibia (white arrow) and an insufficiency fracture line (thin black arrow). C Sagittal T2-weighted image (TR: 4000, TE: 96) shows the insufficiency fracture (arrow)

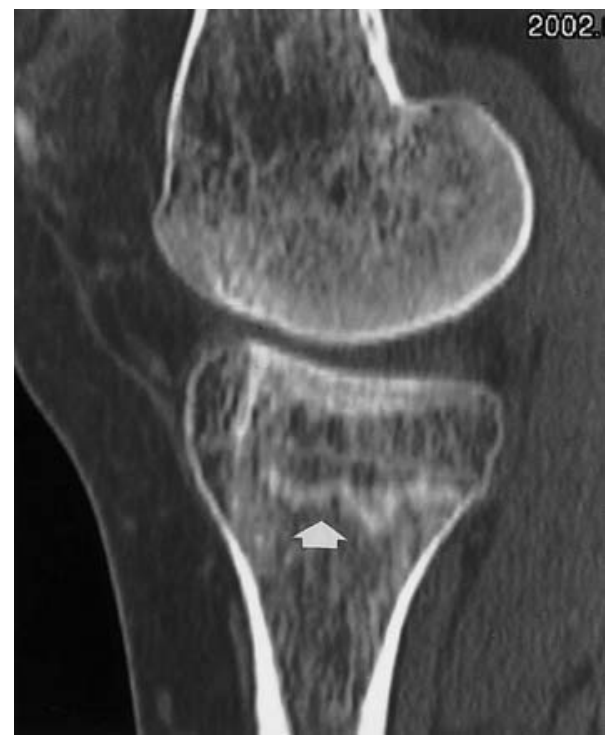

Fig. 3 Computed tomographic sagittal reformatted image shows again the insufficiency fracture (arrow)

nine ratio. There were no signs of infection or malignancy. Underlying pathologies such as osteomalacia were excluded.

Initial radiographs of the knee were normal. Because of persisting knee pain MR imaging was performed and showed an insufficiency fracture of the right proximal tibial plateau associated with a large area of altered marrow signal. There was no evidence of fracture displacement (Fig. 1). A second radiograph of the knee was performed 3 months later, which showed a region of sclerosis highly suggestive of a fracture of the proximal tibial plateau (Fig. 2). CT was performed to exclude the rare possibility of osteoid osteoma associated with a stress fracture, as requested by the consulted orthopaedic surgeon (Fig. 3). The patient was then treated 
with calcium $500 \mathrm{mg}$ daily for 6 months and 10 weeks on crutches with non-weight-bearing. The patient is currently doing well.

\section{Discussion}

Insufficiency fractures are common in joggers [16], military recruits, the elderly, postmenopausal women, and patients with osteopenia due to use of steroids, metabolic or endocrine causes $[14,15]$.

Bushsbaum [12] noted as a possible cause of stress fractures in the pelvic region that during pregnancy the endocrinological balance is changed, causing an alteration of the connective tissue that results in ligament laxity and delayed clinical healing. Moran [11] states that ligament laxity due to hormonal changes in pregnancy and, therefore, muscular stress action in untrained patients, can result in stress/fatigue fractures particularly during delivery. Pavlov et al. [9] showed in their study that joggers with stress fractures all had ligament laxity. Jogging or other sports activity or ligamentous laxity does not appear to be the etiology in our case. Other possible mechanisms of postpartum osteoporosis include secondary bone loss due to prolonged bed rest or the use of magnesium sulfate tocolysis [13], or merely factors related to pregnancy and to lactation [8]. However, the exact cause remains somewhat speculative. It is assumed that metabolic and endocrine changes in pregnancy are responsible for the development of stress fracture [13]. The metabolic changes of pregnancy and lactation relate to the production of PTH-like peptide during this period [14]. With lactation and with the production of PTH-like peptide there is increased bone resorption and, with decreased nutritional calcium intake, there was a negative calcium balance in our patient reflected in her low urinary calcium. This led to a stress fracture.

Our case is interesting because our patient's only risk factor was her postpartum lactating state. While secondary causes of bone loss could be ruled out, pregnancy and lactation associated with low calcium intake were presumed to be the underlying mechanism. Low urinary calcium excretion and a blood phosphate level at the upper limit of normal are a typical constellation during lactation. On the assumption that bone loss during pregnancy and lactation is self-limiting, BMD should increase after nursing is stopped, which could explain why the BMD values of our patient were only slightly diminished at the time of measurement. The MR appearance showed an altered bone marrow signal in the proximal medial tibia and a fracture line. In the absence of a history of trauma, postpartum insufficiency fracture of the proximal tibia was diagnosed.

The patient's tibial stress fracture was treated as a postpartum insufficiency stress fracture with oral calcium medication for 6 months and conservative non-weightbearing for 10 weeks.

\section{References}

1. Hoang TA, Nguyen TH, Daffner RH, Lupetin AR, Deeb ZL. Case report 491. Stress fracture of the right sacrum. Skeletal Radiol 1988; 17:364-367.

2. Schmid L, Pfirrmann C, Hess T, Schlumpf U. Bilateral fracture of the sacrum associated with pregnancy: a case report. Osteoporos Int 1999; 10:91-93.

3. Thienpont E, Simon JP, Fabry G. Sacral stress fracture during pregnancy: a case report. Acta Orthop Scand 1999; 70:525-526.

4. Mikawa Y, Watanabe R, Yamano Y, Miyake S. Stress fracture of the body of the pubis in a pregnant woman. Case report. Arch Orthop Trauma Surg 1988; 107:193-194.

5. Billey T, Dromer C, Pages M, Caulier M, Lassoued S, Fournie B. Spontaneous fracture of the femoral neck in hip algodystrophy in pregnancy. A propos of a case and review of the literature. Rev Rhum Mal Osteoartic 1992; 59:494-496.
6. Knaepler H, Tamm J. Spontaneous fractures of the neck of the femur occurring in pregnancy in the absence of pregnancy osteoporosis. Unfallchirurgie 1984; 10:96-99.

7. Baitner AC, Bernstein AD, Jazrawi AJ, Della Valle CJ, Jazrawi LM. Spontaneous rib fracture during pregnancy. A case report and review of the literature. Bull Hosp Joint Dis 2000; 59:163-165.

8. Gruber HE, Gutteridge DH, Baylink DJ. Osteoporosis associated with pregnancy and lactation: bone biopsy and skeletal features in three patients. Metab Bone Dis Rel Res 1984; 5:159-165.

9. Pavlov H, Nelson TL, Warren RF, Torg JS, Burstein AH. Stress fractures of the pubic ramus. A report of twelve cases. J Bone Joint Surg Am 1982; 64:10201025.

10. Wattanawong $\mathrm{T}$, Wajanavisit $\mathrm{W}$, Laohacharoensombat W. Transient osteoporosis with bilateral fracture of the neck of the femur during pregnancy: a case report. J Med Assoc Thai 2001; 84 [Suppl 2]:516-519.
11. Moran JJ. Stress fractures in pregnancy. Am J Obstet Gynecol 1988; 158:12741277.

12. Bushsbaum HJ. Healing of experimental fractures during pregnancy. Obstet Gynecol 1970; 35:613-615.

13. Levav AL, Chan L, Wapner RJ. Longterm magnesium sulfate tocolysis and maternal osteoporosis in a triplet pregnancy: a case report. Am J Perinatol 1998; 15:43-46.

14. Lippuner K, Zehnder HJ, Casez JP, Takkinen R, Jaeger P. PTH-related protein is released into the mother's bloodstream during lactation: evidence for beneficial effects on maternal calcium-phosphate metabolism. J Bone Miner Res 1996; 11:1394-1399.

15. Reid IR, Wattie DJ, Evans MC, Budayr AA. Post-pregnancy osteoporosis associated with hypercalcaemia. Clin Endocrinol (Oxf) 1992; 37:298-303.

16. McFarland EG, Giangarra C. Sacral stress fractures in athletes. Clin Orthop 1996; 329:240-243. 